\title{
Formación de fase sigma en uniones soldadas de acero inoxidable súper dúplex fundido(*)
}

\author{
J.L. Garin*, R. L. Mannheim* y M.A. Camus**
}

\begin{abstract}
Resumen
El presente trabajo describe las características microestructurales de uniones soldadas de acero súper dúplex fundido (J93404), al ser sometidas a procesos de recocido para inducir la formación de fase sigma a altas temperaturas. Se analizó la influencia del tiempo de calentamiento a $1.073 \mathrm{~K}, 1.123 \mathrm{~K}$ y $1.173 \mathrm{~K}$ sobre la precipitación de fase sigma en la zona afectada térmicamente, metal base y zona de fusión de los conjuntos soldados. Los resultados experimentales evidenciaron la formación de este compuesto intermetálico por descomposición de la fase ferrítica en austenita y sigma. Al comienzo de la transformación la fase nuclea y crece rápidamente en los bordes de grano austenita-ferrita, extendiéndose luego masivamente hacia el seno de la zona ferrítica, con mayor efectividad en términos del aumento de la temperatura de proceso. La formación de sigma en todas las uniones soldadas obedece a un mecanismo de nucleación y crecimiento del tipo Jonson-Mehl-Avrami.
\end{abstract}

\section{Sigma-phase formation in weldments of cast super duplex stainless steel}

\begin{abstract}
This paper decribes the microstructural characteristics of weldments of cast super duplex stainless steel (J93404), being subjected to annealing processes to induce formation of sigma-phase at high temperatures. The influence of heating time at $1073 \mathrm{~K}, 1123 \mathrm{~K}$ and $1173 \mathrm{~K}$ upon precipitation of sigma in the heat affected zone, base metal and fusion zone of the weldments was analyzed. The experimental results revealed the formation of this intermetallic compound throughout decomposition of the ferritic phase into austenite and sigma. At earlier stages of the transformation the phase rapidly nucleates and growth along the ferrite-austenite grain boundaries, and then massively advances towards the bulk of the ferritic zone with greater effectiveness as temperature increases. The formation of sigma-phase in all weldments resembles the Johnson-Mehl-Avrami's mechanism stated for nucleation and growth.
\end{abstract}

Keywords

Sigma-phase; Cast super duplex steel; Microstructure; Casting; Welding.

\section{INTRODUCCIÓN}

El extenso uso que los aceros inoxidables del tipo dúplex han encontrado en la industria moderna, es una consecuencia de su excelente resistencia al agrietamiento por corrosión bajo tensiones en atmósferas cloradas, resistencia a la corrosión localizada y alta resistencia mecánica a la tracción. Tales propiedades posibilitan una gran variedad de aplicaciones industriales, particularmente en el campo de los combustibles, petroquímica, pulpa y papel, generación de energía, transporte marítimo, minería y contaminación ambiental ${ }^{[1}$ y 2$]$. Estas aleaciones presentan una estructura mixta compuesta por cantidades relativas aproximadamente iguales de las fases austenita (estructura FCC) y ferrita (estructura BCC), lo cual se logra mediante un adecuado balance de los elementos aleantes. Las aleaciones de mayores aplicaciones industriales contienen de 22 a $26 \%$ de $\mathrm{Cr}$, de 4 a $7 \%$ de Ni, hasta $4,5 \%$ de $\mathrm{Mo}, 0,7 \%$ de $\mathrm{Cu}$ y W y de 0,08 a $0,35 \%$ de $\mathrm{N}$, además de incluir un bajo contenido de $\mathrm{C}$ (menos de 0,03\%). De esta forma, los aceros inoxidables de mayor resistencia a la corrosión se clasifican como aceros súper dúplex, debido a que exhiben un valor equivalente de resistencia a la corrosión localizada (ERP) sobre 40. El ERP es una

(•) Trabajo recibido el día 12 de Julio de 2010 y aceptado en su forma final el día 8 de Febrero de 2011

* Departamento de Ingeniería Metalúrgica, Universidad de Santiago de Chile, Casilla 10233, Santiago, Chile.

** Departamento de Ingeniería Mecánica, Universidad de Antofagasta, Av. Angamos, 601, Antofagasta, Chile. 
cifra empírica que se utiliza para estimar la resistencia del material a la corrosión localizada en ambientes clorados, calculándose mediante la expresión ${ }^{[3]}$ :

$$
\begin{gathered}
\mathrm{ERP}_{\mathrm{N}}=\% \text { (peso) } \mathrm{Cr}+3,3 \times \% \text { (peso) } \\
\text { Mo }+16 \times \% \text { (peso) } \mathrm{N}
\end{gathered}
$$

En el amplio rango de procesos de manufactura que utilizan aceros dúplex forjados y fundidos, la soldadura de fusión ha significado un rol de gran importancia ${ }^{[4-6]}$. En este contexto, el comportamiento de los aceros dúplex puede ser significativamente afectado por el proceso de soldadura ${ }^{[7-9]}$. Por otra parte, el contenido y la naturaleza de los elementos aleantes, principalmente cromo y molibdeno, así como también la presencia de una matriz ferrítica aumentan la susceptibilidad a la fragilización cuando estos materiales se exponen a altas temperaturas (813-1.173 K) o, en su defecto, a enfriamientos lentos en su proceso de manufactura o por tratamientos térmicos ${ }^{[10-12]}$. Sin embargo, los aceros dúplex no se emplean en aplicaciones en las que la temperatura de servicio sea superior a $573 \mathrm{~K}$ ni suelen someterse a tratamientos térmicos. El propósito de calentarlos a esas temperaturas en el presente trabajo, es investigar el comportamiento de estos materiales en condiciones diversas. El fenómeno de fragilización debe su causa a la precipitación de fases intermedias, principalmente la denominada fase sigma $(\sigma)^{[13]}$, la cual consiste en un compuesto íntermetálico de hierro y cromo con contenidos menores de molibdeno. Esta especie se caracteriza estructuralmente mediante una celda tetragonal tipo Pearson tP30, relacionada a una estequiometría ideal $\mathrm{AX}_{2}{ }^{[14]}$. Sin embargo, su fórmula química efectiva en las aleaciones $\mathrm{Fe}-\mathrm{Cr}$ es aproximadamente $\mathrm{Fe}_{7} \mathrm{Cr}_{6}$, lo cual origina una distribución desordenada de los átomos de hierro y cromo en las posiciones equivalentes del grupo espacial P42/mnm, con factores de ocupación menores que 1,0; esto define un arreglo de poliedros del tipo Frank-Kasper ${ }^{[15}$ y 16]. La complejidad de dicha distribución atómica involucra un gran número de enlaces metálicos fuertemente direccionales, lo cual causa la marcada rigidez del arreglo cristalino y, de esta forma, su nula capacidad de deformación. Los efectos de los elementos aleantes sobre la formación de fase sigma en aceros dúplex son tales que el incremento del contenido relativo de cromo y molibdeno causa un aumento en la rapidez de precipitación y en la máxima cantidad formada; por el contrario, un incremento en el contenido de niquel disminuye la cantidad máxima de sigma que se puede formar, a la vez que acelera el mecanismo de precipitación. Se debe considerar también igual- mente importante como causa de fragilización en aceros dúplex y súper dúplex, la descomposición espinodal que ocurre alrededor de los $748 \mathrm{~K}$, donde la ferrita $\delta$ se transforma en fase $\delta$ rica en hierro y fase $\alpha$ ' rica en cromo. Las principales fases que se encuentran en el sistema ternario en estudio son las soluciones sólidas ferrita $(\mathrm{Fe}, \mathrm{Cr}, \mathrm{Ni})$, austenita $(\mathrm{Fe}, \mathrm{Cr}, \mathrm{Ni})$ y la fase intermedia sigma $\left(\mathrm{Fe}_{7} \mathrm{Cr}_{6}\right)$. También se pueden formar otras fases secundarias, tales como $\alpha^{\prime}, \mu, \chi, \eta, \varepsilon$ y $\mathrm{G}^{[7]}$, pero, con la excepción de $\chi^{[17]}$, su detección experimental es normalmente muy difícil debido a sus bajas concentraciones en la aleación. La formación de fase sigma puede ser también muy perjudicial en muchas aplicaciones de los aceros dúplex, debido a que permite una alta penetración de la corrosión ${ }^{[18-21]}$. En términos generales, basta la formación de una pequeña cantidad relativa de este compuesto para reducir fuertemente la ductilidad, tenacidad y resistencia a la corrosión localizada de las uniones soldadas de aceros dúplex ${ }^{[22]}$.

Desde el punto de vista del proceso de fabricación los aceros súper dúplex fundidos son ampliamente utilizados en medios acuosos a temperatura ambiente y en contacto con gases y líquidos calientes hasta $573 \mathrm{~K}$. Comparados con los aceros dúplex ordinarios, exhiben mayor resistencia mecánica y resistencia a la corrosión, particularmente corrosión localizada y corrosión bajo tensiones en ambientes clorados ${ }^{[23]}$. Sin embargo, dada la presencia y concentración de los elementos cromo y molibdeno en este grupo de aceros inoxidables, estos son muy susceptibles a la formación de fase sigma. En virtud de estas consideraciones el presente trabajo centra su atención en la formación de fase sigma en uniones soldadas de un acero de grado súper dúplex, en términos de calentamiento a altas temperaturas durante diferentes periodos de tiempo. Para efectos del estudio se seleccionó una aleación fundida J93404, clasificada por la norma ASTM A 890 [24].

\section{PROCEDIMIENTO EXPERIMENTAL}

El desarrollo experimental de este trabajo utilizó técnicas usuales de procesamiento y análisis de materiales, como soldadura de fusión, tratamientos térmicos para precipitación de fase sigma, caracterización química y microestructural, difracción de rayos X, microscopía electrónica de barrido con microanálisis y mediciones magnéticas. 


\subsection{Aleación base y soldadura}

La aleación fundida utilizada como material base en esta investigación fue fabricada por un proveedor comercial y entregada en la forma de probetas tipo "Y", con sección de ensayo de $50 \mathrm{~mm}$ de espesor, de acuerdo a la norma ASTM A395. La composición química de las piezas $Y$, determinada mediante espectrometría visible de emisión (SPECTROLAB), se detalla en la tabla I. Los valores obtenidos se encuentran dentro de los rangos de composición establecidos por la norma ASTM A 890/A 890M grado 5a (UNS J93404) para los aceros fundidos del tipo súper dúplex. El contenido de molibdeno es ligeramente inferior al de referencia, siendo compensado con adición de cobre para efectos de una mejor resistencia a la corrosión. Esta composición química permite calcular el valor del equivalente de resistencia a la corrosión localizada de la aleación, de acuerdo a la expresión (1):

$$
\begin{gathered}
\mathrm{ERP}_{\mathrm{N}}=24,7+3,3 \times 3,74+ \\
16 \times 0,25=41,04
\end{gathered}
$$

Las muestras para manufactura de las uniones soldadas se extrajeron de diferentes niveles de las secciones de prueba de los bloques Y, con un espesor de $15 \mathrm{~mm}$. La soldadura se efectuó mediante la técnica de arco manual (SMAW), corriente de trabajo 70 amperios, tensión de trabajo 22 voltios y velocidad de soldeo 10 ( $\mathrm{cm} / \mathrm{min})$; con estos parámetros de operación es posible determinar el aporte térmico por medio de la ecuación (2):

$$
\begin{gathered}
\mathrm{H}=\mathrm{V}(\text { voltaje }) \times I(\text { intensidad }) / \\
\mathrm{v}(\text { velocidad de soldeo })
\end{gathered}
$$

La aplicación de esta ecuación resulta en un valor de $0,92(\mathrm{KJ} / \mathrm{mm})$, el cual se encuentra en el rango de 0,5 a $2(\mathrm{KJ} / \mathrm{mm})$ establecido por los códigos correspondientes.

La soldadura de las uniones se efectuó en la modalidad de "tope" con bisel tipo "X", multi depósito del material de aporte, con siete pasadas y temperatura de inter pasada de $393 \mathrm{~K}$. Para estos efectos se hizo uso del electrodo INOX 2509MoB, clasificado como E2594-15 según la norma AWS SFA5.4. La composición típica de este electrodo se presenta en la tabla II. Con el propósito de estimar el alcance del fenómeno de dilución en la zona de fusión, se consideraron los contenidos equivalentes de cromo y níquel del metal base y del metal de aporte (electrodo), los cuales fueron calculados mediante las siguientes ecuaciones, establecidas por la norma

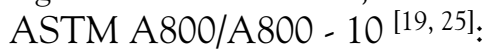

$$
\begin{gathered}
\mathrm{Cr}_{\text {eq }}=\% \mathrm{Cr}+1,5 \times \% \mathrm{Si}+1,4 \times \\
\% \mathrm{Mo}+\% \mathrm{Nb}-4,99 \\
\mathrm{Ni}_{\text {eq }}=\% \mathrm{Ni}+30 \times \% \mathrm{C}+0,5 \times \\
\% \mathrm{Mn}+26 \times(\% \mathrm{~N}-0,02)+2,77
\end{gathered}
$$

donde las concentraciones indicadas se expresan en porcentaje en peso. La aplicación de dichas ecuaciones a los materiales utilizados entrega los siguientes valores:

Tabla I. Composición química de la aleación (\% en peso)

Table I. Chemical composition of the alloy (weight \%)

\begin{tabular}{ccc}
\hline Elemento & Muestra $^{\mathbf{a}}$ & Norma $^{\mathbf{b}}$ \\
\hline $\mathrm{C}$ & 0,026 & 0,03 \\
$\mathrm{Cr}$ & 24,70 & $24,0-26,0$ \\
$\mathrm{Ni}$ & 6,45 & $6,0-8,0$ \\
$\mathrm{Mo}$ & 3,74 & $4,0-5,0$ \\
$\mathrm{Cu}$ & 0,12 & - \\
$\mathrm{N}$ & 0,25 & $0,10-0,30$ \\
$\mathrm{Mn}$ & 0,92 & 1,50 \\
$\mathrm{Si}$ & 0,57 & 1,00 \\
$\mathrm{P}$ & 0,03 & 0,04 \\
$\mathrm{~S}$ & 0,007 & 0,04 \\
\hline
\end{tabular}

a: promedio de tres mediciones por muestra.

b: valores simples indican contenido máximo. 
Tabla II. Composición típica del metal de aporte (\% en peso)

Table II. Typical weld metal composition (weight \%)

\begin{tabular}{ccccccccc}
\hline $\mathbf{C}$ & $\mathbf{S i}$ & $\mathbf{M n}$ & $\mathbf{C r}$ & $\mathbf{N i}$ & $\mathbf{M o}$ & $\mathbf{C u}$ & $\mathbf{N}$ & $\mathbf{F e}$ \\
\hline$<0,04$ & 0,5 & 1,5 & 25,0 & 9,5 & 4,0 & 0,7 & 0,23 & base \\
\hline
\end{tabular}

metal base

$$
\begin{gathered}
\mathrm{Cr}_{\mathrm{eq}}=24,70+1,5 \times 0,57+ \\
1,4 \times 3,74-4,99=25,80 \\
\mathrm{Ni}_{\mathrm{eq}}=6,45+30 \times 0,026+0,5 \times 0,92+ \\
26 \times(0,25-0,02)+2,77=16,44
\end{gathered}
$$

metal de aporte

$$
\begin{gathered}
\mathrm{Cr}_{\mathrm{eq}}=25,00+1,5 \times 0,50+ \\
1,4 \times 4,00-4,99=26,36 \\
\mathrm{Ni}_{\mathrm{eq}}=9,50+30 \times 0,04+0,5 \times 1,50+ \\
26 \times(0,25-0,02)+2,77=20,02
\end{gathered}
$$

\subsection{Metalografía y tratamientos térmicos}

Las probetas para observación metalográfica y tratamientos térmicos de precipitación de sigma se extrajeron de las uniones soldadas, logrando una superficie de trabajo rectangular de $15 \times 45 \mathrm{~mm}^{2}$ y $5 \mathrm{~mm}$ de espesor, asegurando, así, la presencia del cordón de soldadura (CS), zona afectada térmicamente (ZAT) y metal base (MB). Los recocidos de dichas uniones soldadas para inducir la formación de fase sigma se efectuaron a $1.073 \mathrm{~K}, 1.123 \mathrm{~K}$ y $1.173 \mathrm{~K}$, durante periodos de tiempo desde 3 hasta $96 \mathrm{~h}$, finalizando cada calentamiento con enfriamiento en agua a temperatura ambiente. La caracterización microestructural cuantitativa de todas las probetas procedió por medio de microscopía de luz (ZEISS-AXIOTECH) y procesamiento digital de imágenes (KS-PHASE). Para estos efectos se procedió de acuerdo a la norma ASTM E-3, considerando cortes transversales y paralelos a la superficie de prueba del bloque Y. Cada medida se realizó en el centro, medio centro y borde de la superficie bajo observación, lo cual determina un total de seis mediciones por probeta. Las superficies a observar se prepararon mediante técnicas normales de lijado, para terminar con pulidos sucesivos en alúmina de
$5,0,3$ y $0,05 \mu \mathrm{m}$. Para revelar la microestructura a rasgos generales se utilizó el reactivo de Vilella ${ }^{\text {[26] }}$ durante 30 s de ataque, mientras que la observación de la fase sigma fue posible mediante ataque electrolítico con solución de $\mathrm{KOH} 10 \mathrm{~N}$, bajo una tensión de 3 voltios y 4 s de inmersión. Finalmente, la ausencia de posibles carburos de hierro y cromo se aseguró mediante el uso de solución de $\mathrm{NH}_{4}$ al 28-30 \% vol. Con el propósito de visualizar la morfología fina de los precipitados obtenidos, la observación metalográfica se complementó mediante microscopía electrónica de barrido (JEOL 5410) y microanálisis EDS (NORAN-Vantage 1.4.2) para determinar el contenido químico de cada fase.

\subsection{Difracción de rayos $X$}

La identificación estructural de las fases componentes en cada probeta hizo uso de técnicas de difracción de rayos X para agregados policristalinos. Se utilizó un difractómetro SIEMENS D5000 equipado con monocromador de grafito para el haz difractado, radiación $K \alpha 1$ de cobre $(\lambda=0,15406 \mathrm{~nm}, 40 \mathrm{kV}$, $30 \mathrm{~mA}$ ), rendija de divergencia de $1 \mathrm{~mm}$, rendija de dispersión de $1 \mathrm{~mm}$ y rendija receptora de $0,6 \mathrm{~mm}$, geometría de Bragg-Brentano y modalidad de barrido $\theta-\theta$; el rango de barrido se fijó en $30-85^{\circ}$, con un paso de $0,02^{\circ}$ y tiempo de medición de $10 \mathrm{~s}$ por paso. El instrumento operó bajo el sistema de programas DIFFRAC-Plus para adquisición y procesamiento de los datos experimentales. Cabe destacar, finalmente, que esta técnica de análisis se utilizó únicamente como herramienta cualitativa para identificar estructuralmente la presencia de las fases en estudio.

\subsection{Mediciones magnéticas}

El contenido relativo de ferrita en las probetas tratadas térmicamente se determinó por medio de ferritoscopía (FISCHER MP30E-S), aportando, de esta manera, valores de gran exactitud para comparación con aquellos determinados mediante metalografía 
cuantitativa. La calibración del instrumento se efectuó mediante patrones WRC 10/80 para FN (ferrite number o número de ferrita) y \% de Fe. Se utilizó una punta de prueba de $0,5 \mathrm{~mm}$ de diámetro, lo cual hizo posible la medición de ferrita sin interferencia del metal base ni del metal de aporte, siendo este hecho corroborado por medio de observaciones metalográficas.

\section{RESULTADOS Y DISCUSIÓN}

\subsection{Formación de fase sigma: microestructura}

La observación metalográfica efectuada sobre la aleación original (metal base) y sobre una muestra de las uniones soldadas sin tratamiento térmico, reveló las microestructuras que se muestran en las figuras 1 y 2 respectivamente. La microestructura de colada que se observa en la figura 1 caracteriza una distribución de granos de austenita (partículas claras) en la matriz de ferrita (fondo obscuro); los granos de austenita presentan en parte una morfología aproximadamente equiaxial, a la vez que en algunas regiones de menor extensión la tendencia de cristalización deriva a una distribución de tipo Widmansttäten. La figura 2 exhibe la microestructura próxima al cordón de soldadura, evidenciando, así, una distribución de partículas de austenita de menor tamaño en la ZAT, y luego una microestructura característica del metal base, donde no han ocurrido mayores efectos derivados del proceso de soldadura. El difractograma de rayos X correspondiente a esta probeta se exhibe en la figura 3, donde se puede identificar la presencia de las dos fases componentes del material y, sin lugar a dudas, la ausencia de fase sigma, cuya cinética de precipitación es relativamente lenta en comparación con el tiempo de enfriamiento de la soldadura, en el rango de las temperaturas en estudio. Otro aspecto importante que se destaca en ese diagrama de difracción, es la fuerte orientación preferencial de los planos de austenita, $\{220\}$, con respecto a los $\{111\}$, lo que indica una texturización del tipo $\{110\}$, inducida por la etapa de solidificación de la soldadura. No se detecto un efecto similar en la fase ferrítica.

Para efectos de una descripción concisa de los resultados microestructurales originados por los tratamientos térmicos, se considerarán tres muestras representativas de cada una de las temperaturas de recocido experimentadas, correspondientes a tiempos de tratamiento de 12,24 y $72 \mathrm{~h}$, con especial atención a la ZAT y a la zona de fusión (cordón de soldadura) en cada caso. Las figuras 4, 5 y 6 ilustran las microestructuras obtenidas mediante recocido de las probetas soldadas, a $1.073 \mathrm{~K}, 1.123 \mathrm{~K}$ y $1.173 \mathrm{~K}$ respectivamente. En la figura 4 a) se observan los granos de austenita como "islotes" con tonalidad clara, a la vez que se destaca una masiva distribución de austenita secundaria $\left(\gamma_{2}\right)$, compuesta por partículas de menor tamaño y forma irregular; esta austenita secundaria precipita como subproducto de la transformación de ferrita en fase sigma

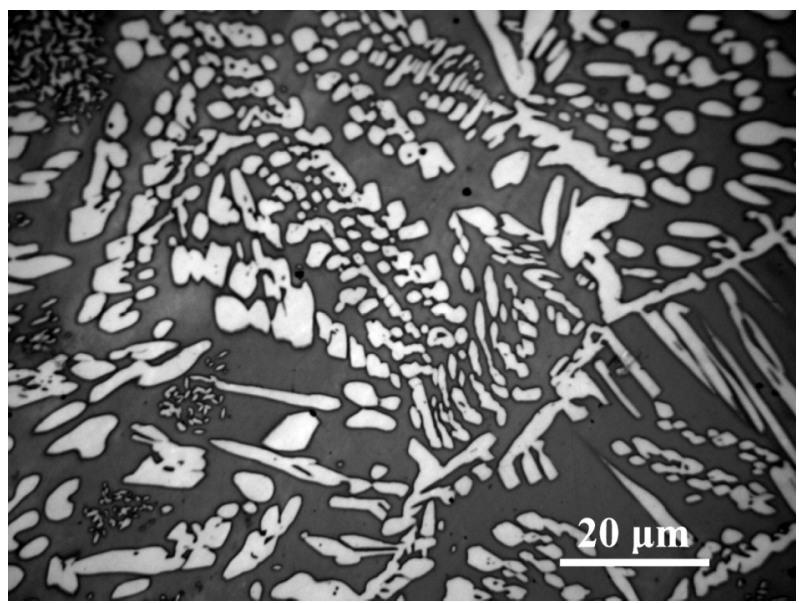

Figura 1. Microestructura de colada de la aleación original. Granos de austenita (partículas claras) sobre matriz de ferrita (fondo oscuro).

Figure 1. As-cast microstructure of the raw alloy. Austenite grains (clear particles) on a ferrite matrix (dark background).

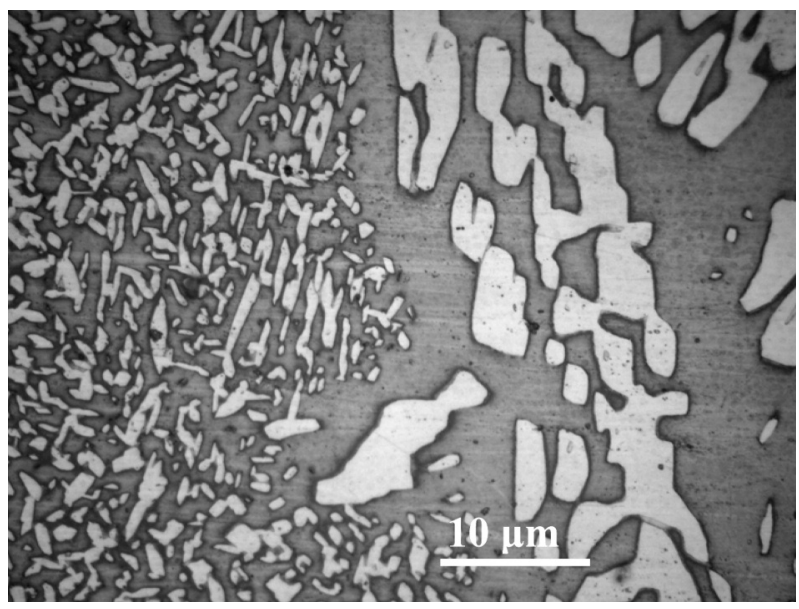

Figura 2. Microestructura de la ZAT y metal base de la unión soldada sin tratamiento térmico.

Figure 2. Microstructure of the HAZ and base metal of the weldment with no heat treatment. 


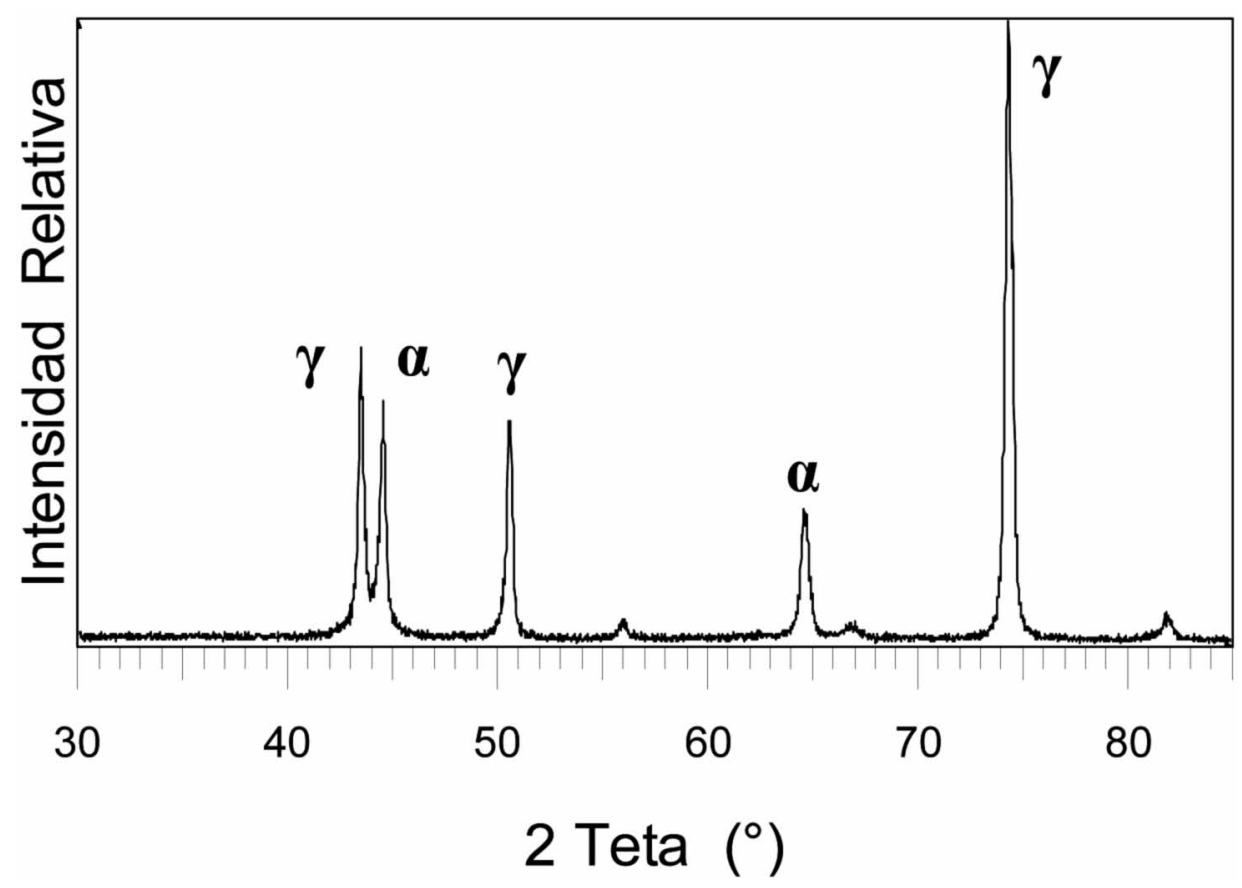

Figura 3. Difractograma de rayos $X$ de la unión soldada, sin tratamiento térmico. El pico a $74,2^{\circ}$ indica una fuerte orientación preferencial de los planos $\{110\}$ de la austenita.

Figure 3. X-ray diffraction pattern of the weldment with no heat treatment. The peak at $74.2^{\circ}$ reveals a strong preferred orientation of the $\{110\}$ planes of austenite.

durante la etapa de recocido . Las partículas de fase sigma se visualizan con un tamaño muy reducido, distribuidas entre los granos de austenita secundaria, sobre un fondo oscuro correspondiente a la ferrita residual, después de completarse la transformación a esa temperatura. La figura 4 b) muestra con mayor detalle la morfología de precipitación de sigma, caracterizada por agrupamiento de partículas de forma aproximadamente equiaxial y también alargada, ocupando lugares entre los granos de austenita secundaria, que previamente fueron zonas de ferrita. Similarmente, las figuras 4 c) y 4 d) exhiben un comportamiento similar en la precipitación de fase sigma, donde se registra una mayor concentración de partículas en términos del mayor tiempo de recocido de las probetas. De igual modo, la serie de figuras 5 y 6 presenta una mejor delineación del fenómeno, donde el parámetro de temperatura de recocido conlleva mayores contenidos relativos y tamaños de partícula de la fase precipitada.

Las fases componentes de cada microestructura fueron finalmente corroboradas mediante pruebas de difracción de rayos X. Para fines demostrativos en el presente artículo se han considerado los diagramas de difracción correspondientes a las ZAT de las probetas procesadas a $1.073 \mathrm{~K}, 1.133 \mathrm{~K} \mathrm{y}$ $1.173 \mathrm{~K}$, durante $72 \mathrm{~h}$. Estos resultados se ilustran en las figuras 7 a), 7 b) y 7 c) respectivamente, donde se identifican preferentemente las líneas de mayor intensidad de cada fase. La formación de fase sigma se comprueba, de esta forma, en todas las probetas sometidas a recocido a las temperaturas y tiempos indicados en el procedimiento experimental.

\subsection{Formación de fase sigma: parámetros de nucleación y crecimiento}

Habiendo dilucidado la morfología y distribución de partículas en el proceso de formación de fase sigma, el tópico de interés de este apartado se centra en los parámetros de temperatura y tiempo asociados a los tratamientos de recocido de las diferentes probetas estudiadas. Así, los resultados de la determinación cuantitativa de esta fase en términos del tiempo de tratamiento a las temperaturas de trabajo, se presentan en los gráficos de las figuras 8 a), 8 b) y 8 c); las correspondientes zonas de análisis en las probetas se han clasificado como ZAT 


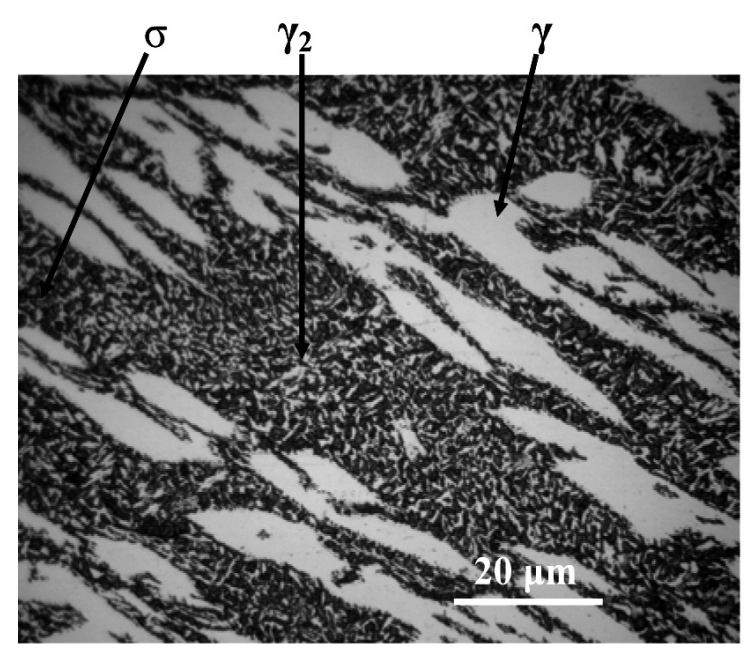

(a)

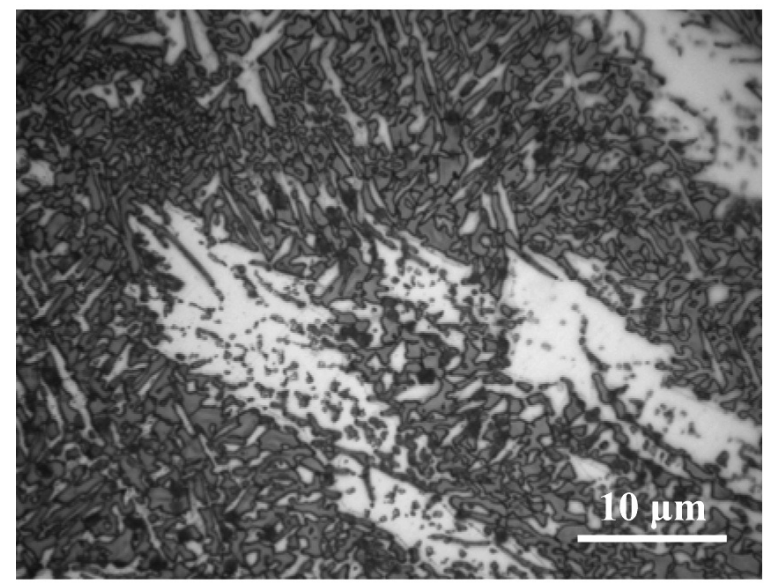

(c)

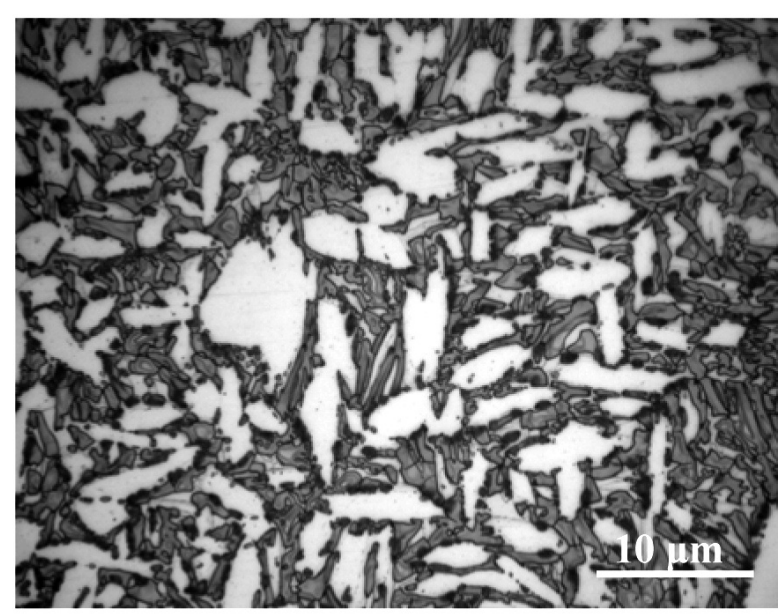

(b)

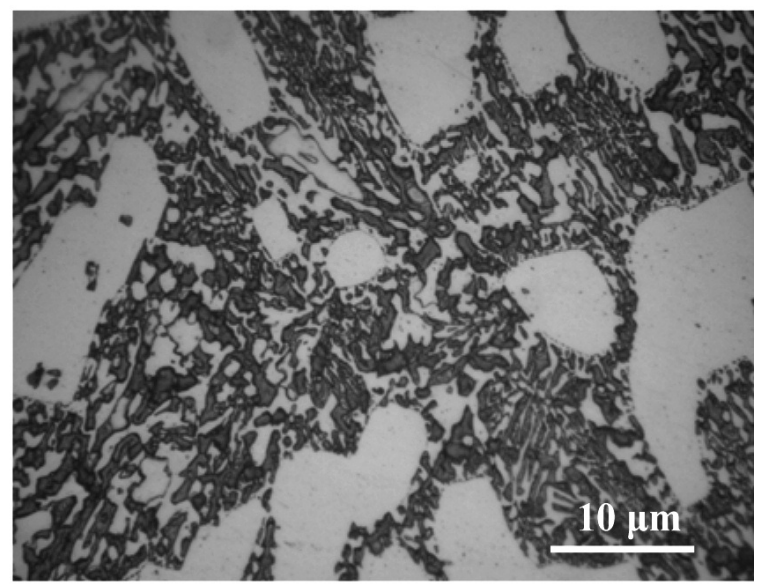

(d)

Figura 4. Microestructura de la unión soldada (ZAT) y recocida a $1.073 \mathrm{~K}$; a) $12 \mathrm{~h}$ de tratamiento, b) Mayor detalle de a), c) $24 \mathrm{~h}$ de tratamiento, d) $72 \mathrm{~h}$ de tratamiento.

\section{Figure 4. Microstructure of the weldment (HAZ) annealed at 1,073 K; a) $12 \mathrm{~h}$ treatment, b) Larger detail of a), c) $24 \mathrm{~h}$ treatment, d) $72 \mathrm{~h}$ treatment.}

(zona afectada térmicamente), ZF zona de fusión) y MB (metal base). El comportamiento de las curvas en todos los casos analizados se puede asimilar al clásico mecanismo de Jonson-Mehl-Avrami, establecido para un proceso de nucleación y crecimiento controlado por difusión ${ }^{[27]}$. En este contexto, la observación de cada uno de los gráficos revela que la rapidez de nucleación y crecimiento de las partículas de fase sigma, a una determinada temperatura, es muy alta para tiempos de tratamiento inferiores a $20 \mathrm{~h}$, a la vez que sobre $40 \mathrm{~h}$ de proceso el fenómeno tiende a alcanzar asintóticamente los valores máximos de concentración de sigma, en cada una de las zonas analizadas. En términos globales la concentración de sigma en la ZAT es mayor que la observada en el metal base, la cual es, a su vez, mayor que aquella encontrada en la zona de fusión. Este efecto es causado por la cantidad relativa de ferrita formada originalmente en cada una de esas zonas, como consecuencia del proceso de soldadura:

Número de Ferrita en la ZAT: 84,00, Número de Ferrita en el MB: 79,33, Número de Ferrita en la ZF: 43,62 .

Dado que la fase ferrítica es el principal componente microestructural que origina la formación de fase sigma, mayores concentraciones derivan en mayores cantidades relativas de sigma. Por su parte, la influencia de la temperatura en la formación de fase sigma favorece la presencia de este componente, como se puede observar mediante comparación de los gráficos presentados en las figuras 8 a), 8b) y 8 c). La causa de este com- 


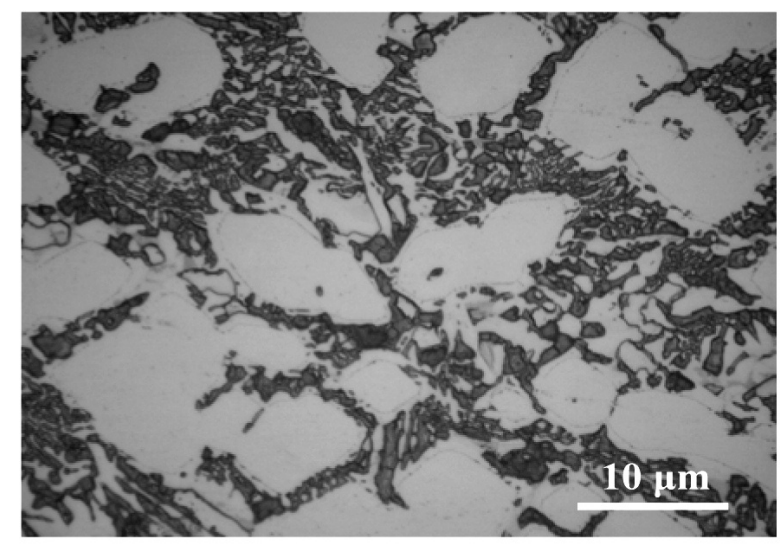

(a)

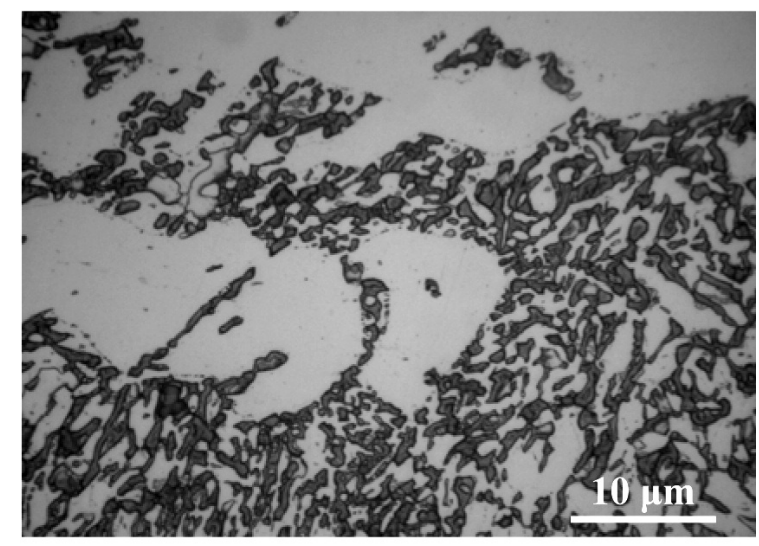

(b)

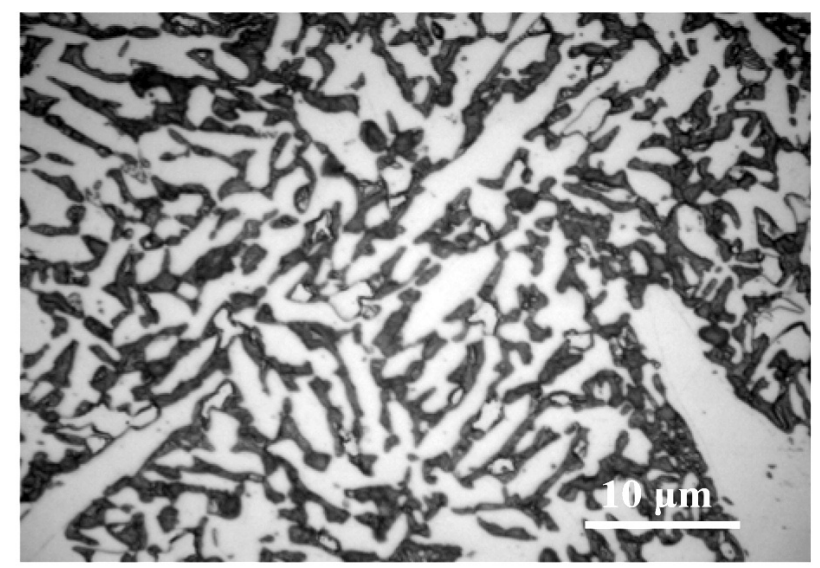

(c)

Figura 5. Microestructura de la unión soldada (ZAT) y recocida a $1.123 \mathrm{~K}$; a) $12 \mathrm{~h}$ de tratamiento, b) $24 \mathrm{~h}$ de tratamiento, c) $72 \mathrm{~h}$ de tratamiento.

Figure 5. Microstructure of the weldment (HAZ) annealed at 1,123 K; a) $12 \mathrm{~h}$ treatment, b) $24 \mathrm{~h}$ treatment, c) $72 \mathrm{~h}$ treatment.

portamiento térmico del fenómeno obedece a la mayor difusividad atómica en la matriz de ferrita, cuando aumenta su temperatura de recocido.

Para efectos de modelación de las curvas bajo los resultados experimentales, se consideró la forma general de la ecuación de Avrami como:

$$
X_{\sigma}=a\left[1-\exp \left(-k t^{n}\right)\right]
$$

donde $\mathrm{X}$ es la fracción transformada de fase sigma y $\mathrm{k}$ y $\mathrm{n}$ son constantes físicamente asociadas al mecanismo de nucleación y crecimiento, propias de cada material y proceso. El ajuste de curvas ligadas a los puntos experimentales de acuerdo a la ecuación (5), se logró mediante la aplicación de una rutina de mínimos cuadrados no lineales (SIGMA PLOT). Los resultados obtenidos se detallan en la tabla III. Los valores del parámetro "a" incluido en esa tabla, indican el contenido teórico máximo de fase sigma que se puede esperar en las condiciones experimentales aplicadas a cada caso.

\subsection{Formación de fase sigma: transformación de la ferrita}

La evolución microestructural de la fase ferritica durante el recocido de las probetas reviste especial interés, por cuanto es el micro constituyente precursor de la fase sigma, a través de la reacción:

$$
\delta \longrightarrow \sigma+\gamma_{2}
$$

De acuerdo a esa ecuación la ferrita delta se transforma completamente, originando así, la formación de fase sigma y austenita secundaria, por lo cual aumenta el contenido relativo de este último 


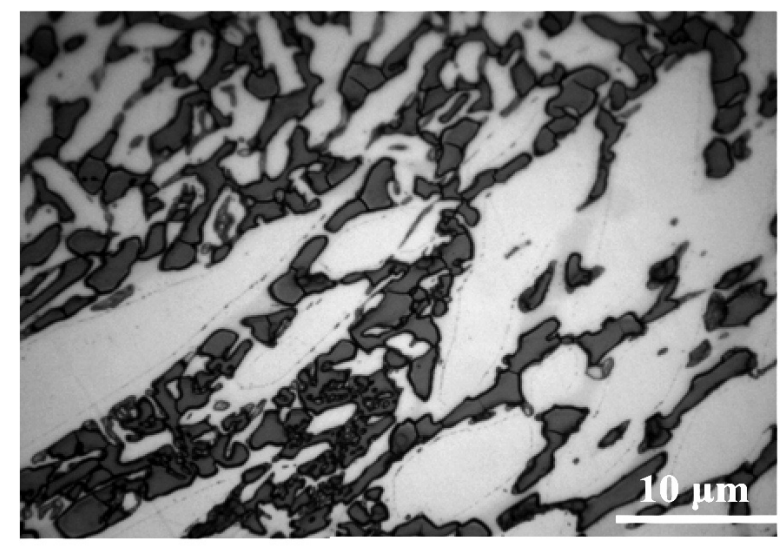

(a)

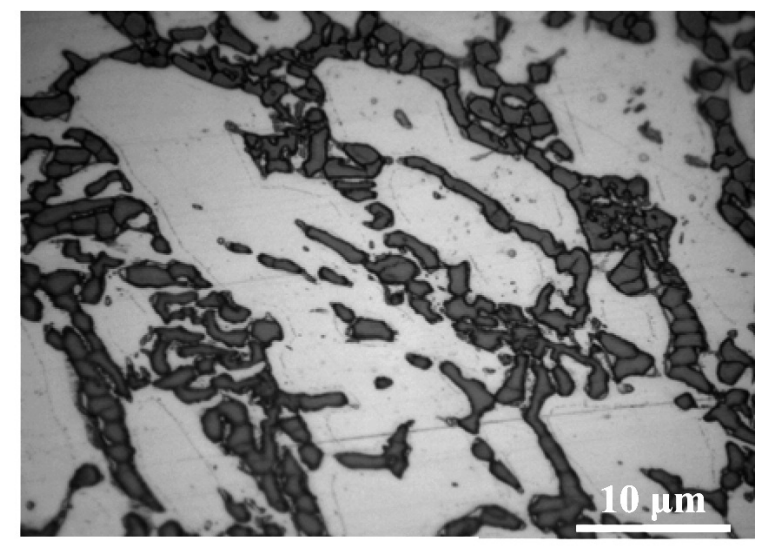

(b)

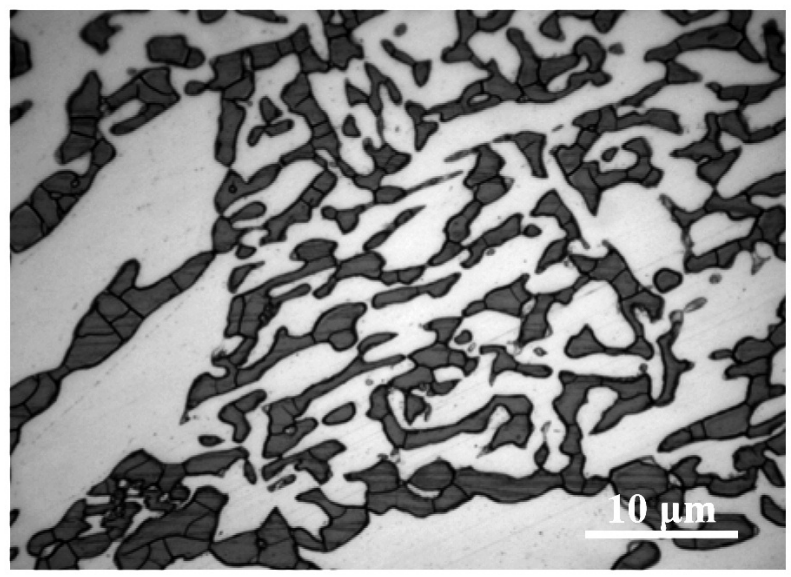

(c)

Figura 6. Microestructura de la unión soldada (ZAT) y recocida a $1.173 \mathrm{~K}$; a) $12 \mathrm{~h}$ de tratamiento, b) $24 \mathrm{~h}$ de tratamiento, c) $72 \mathrm{~h}$ de tratamiento.

Figure 6. Microstructure of the weldment (HAZ) annealed at $1.173 \mathrm{~K}$; a) $12 \mathrm{~h}$ treatment, b) $24 \mathrm{~h}$. treatment, c) $72 \mathrm{~h}$ treatment.

Tabla III. Parámetros de Jonson-Mehl-Avrami

Table III. Jonson-Mehl-Avrami' parameters

\begin{tabular}{cccccc}
\hline Temperatura (K) & Zona & $\mathbf{a}$ & $\mathbf{k}$ & $\mathbf{N}$ & $\mathbf{R}$ \\
\hline \multirow{2}{*}{1.073} & ZAT & 33,22 & 0,39 & 0,60 & 0,98 \\
& MB & 34,69 & 0,47 & 0,36 & 0,99 \\
\multirow{2}{*}{1.123} & ZF & 32,51 & 0,37 & 0,42 & 1,00 \\
& ZAT & 41,54 & 0,37 & 0,43 & 0,99 \\
\multirow{2}{*}{1.173} & MB & 46,35 & 0,33 & 0,34 & 0,99 \\
& ZF & 41,93 & 0,35 & 0,31 & 0,99 \\
& ZAT & 41,53 & 0,44 & 0,63 & 1,00 \\
& MB & 38,64 & 0,44 & 0,71 & 0,99 \\
& ZF & 31,60 & 0,74 & 0,23 & 0,96 \\
\hline
\end{tabular}



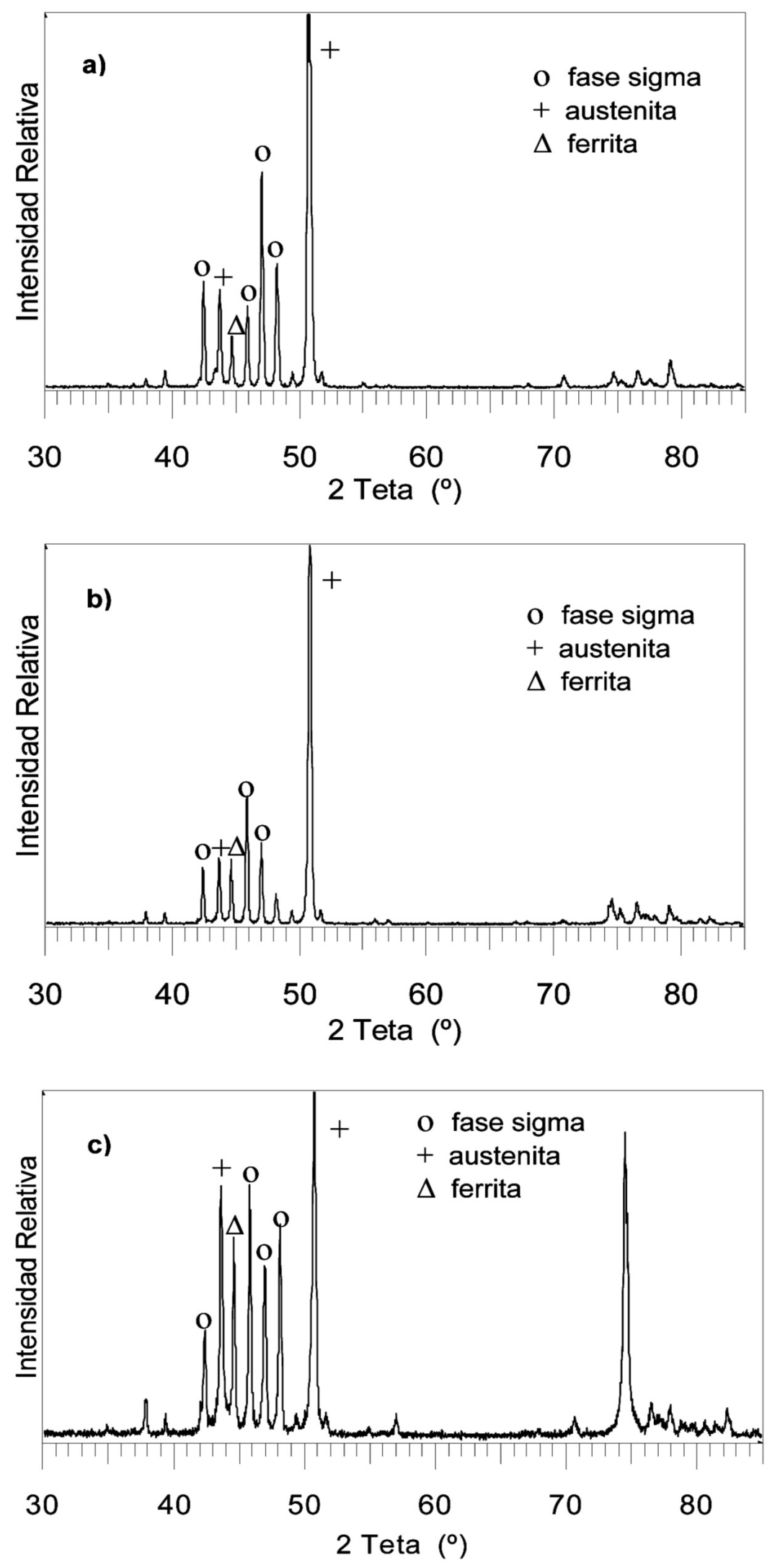

Figura 7. Difractograma de rayos $X$ de la ZAT recocida durante $72 \mathrm{~h} \mathrm{a)} 1.073 \mathrm{~K}, \mathrm{~b}) 1.123 \mathrm{~K}, \mathrm{c}) 1.173 \mathrm{~K}$.

Figure 7. X-ray diffraction pattern of $H A Z$ annealed for $72 h$; a) $1,073 \mathrm{~K}, b) 1,123 \mathrm{~K}, \mathrm{c}) 1,173 \mathrm{~K}$. 


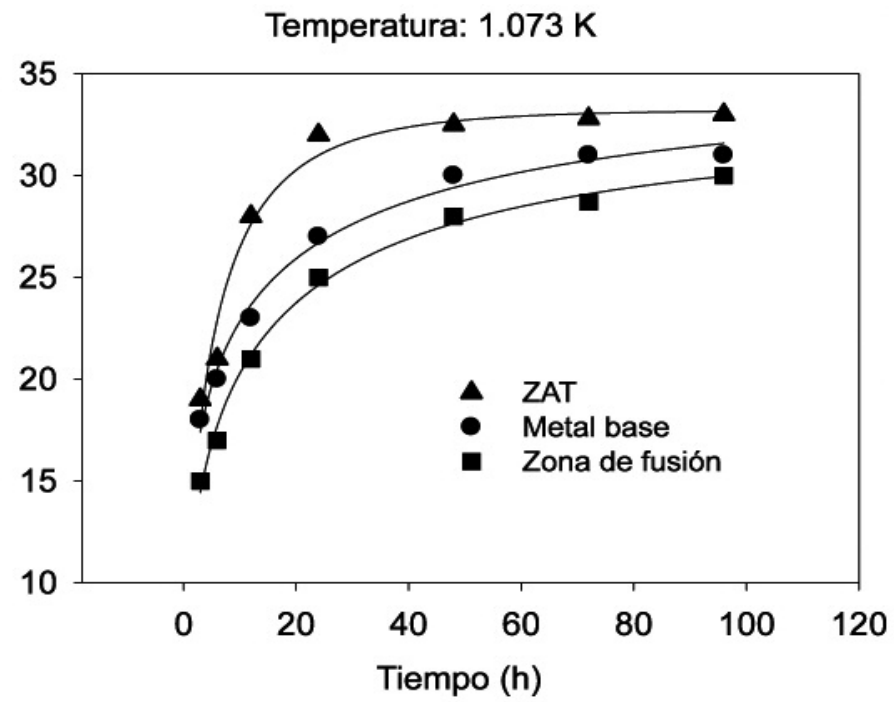

Temperatura: $1.133 \mathrm{~K}$

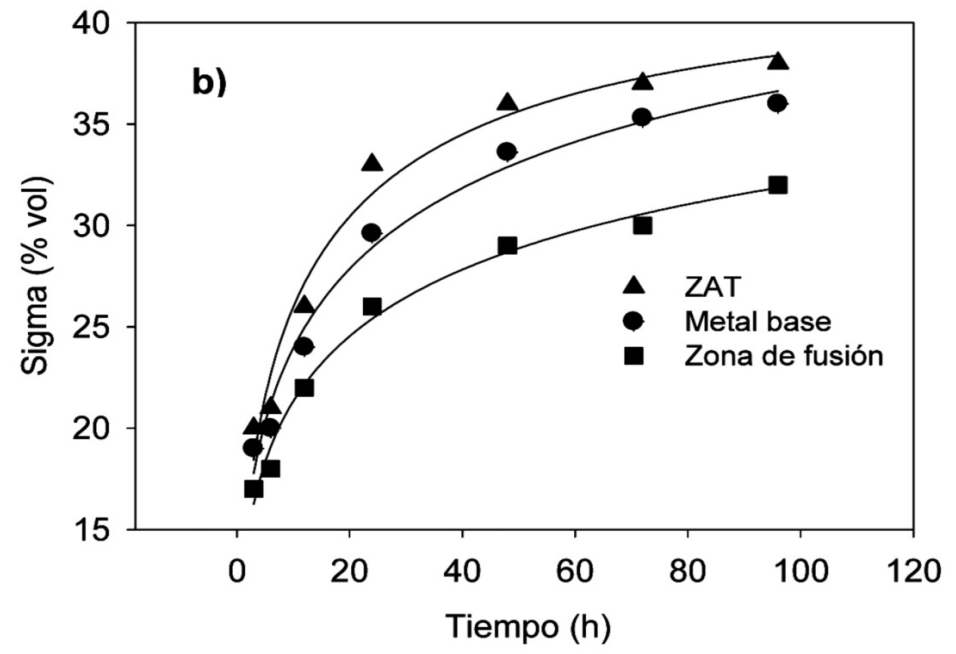

Temperatura: $1.173 \mathrm{~K}$

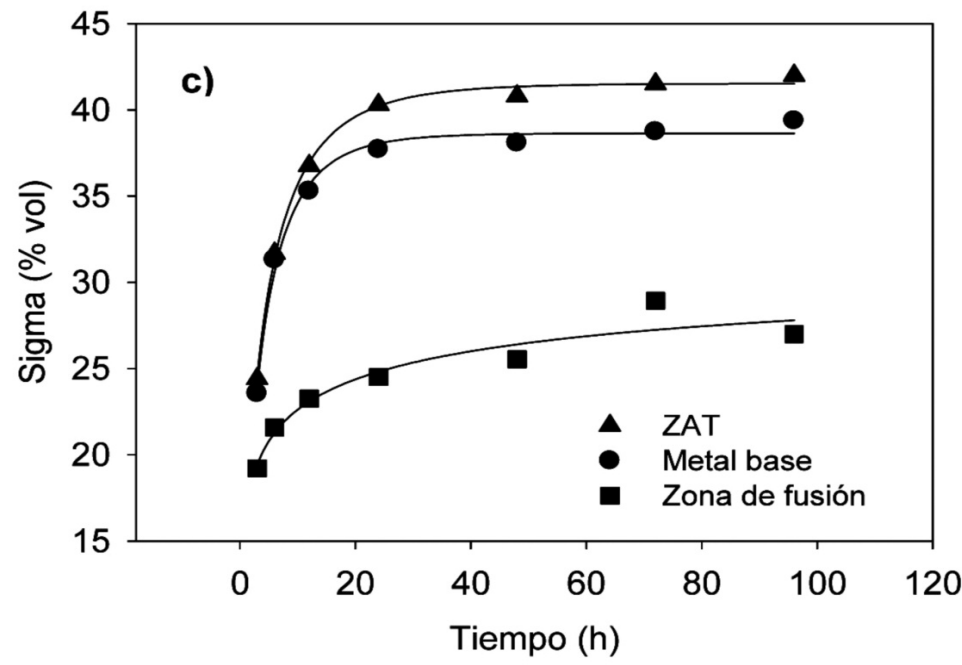

Figura 8. Contenido relativo de fase sigma en la ZAT, MB y ZF en términos del tiempo de calentamiento.

Figure 8. Relative contents of sigma phase in the HAZ, BM and FZ versus heating time. 


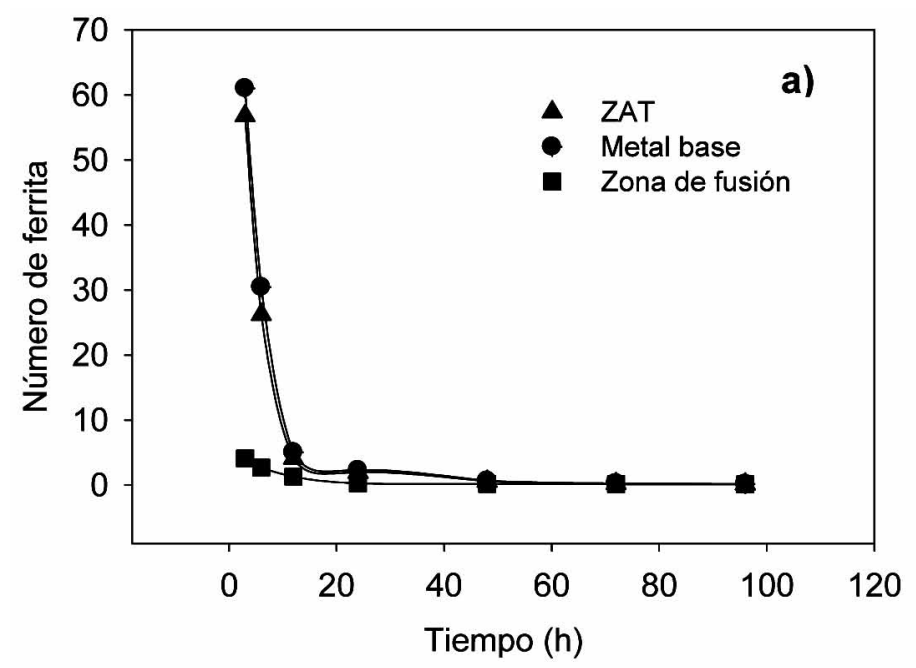

Temperatura: $1.123 \mathrm{~K}$

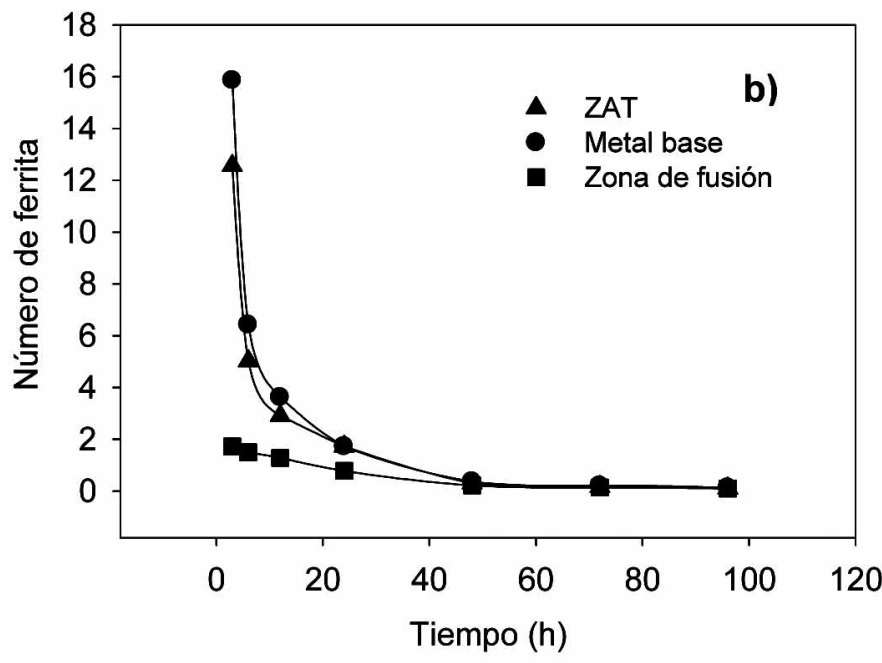

Temperatura: $1.173 \mathrm{~K}$

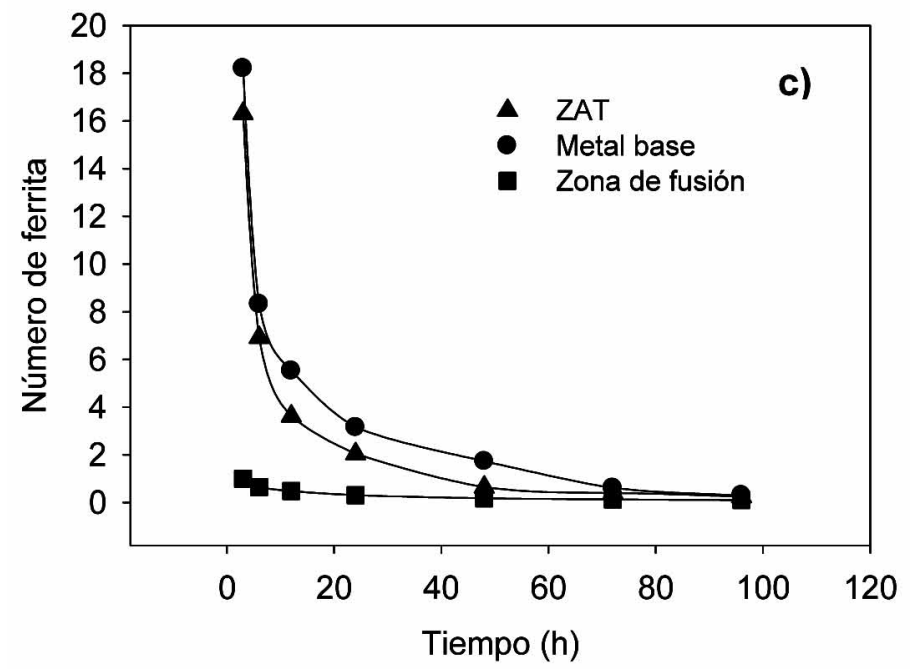

Figura 9. Contenido relativo de ferrita (ferritometría) en la ZAT, MB y ZF en términos del tiempo de calentamiento; a) $1.073 \mathrm{~K}$, b) $1.123 \mathrm{~K}, \mathrm{c}) 1.173 \mathrm{~K}$.

Figure 9. Relative contents of ferrite (feritscope) in the HAZ, BM and $F Z$ versus heating time; a) 1,1073 K, b) 1,123 K, c) 1,173 K. 
componente en la microestructura. La variación del contenido relativo de ferrita en términos del tiempo de recocido se expone gráficamente en las figuras 9 a), 9 b) y 9 c). Se observa que la presencia de la fase ferrítica experimenta un rápido decaimiento en la ZAT y metal base durante las primeras $20 \mathrm{~h}$ de tratamiento, para reducirse a valores inferiores a $1,00 \%(\mathrm{vol})$ cuando el tiempo de recocido excede la cifra señalada. En el caso de la zona de fusión la disminución de ferrita es notoriamente más efectiva, debido al mayor contenido de elementos estabilizadores de austenita en el metal de aporte, tales como níquel y manganeso.

\section{CONCLUSIONES}

Los resultados del presente trabajo aportan mayores conocimientos sobre la formación de fase sigma en uniones soldadas de un tipo de acero súper dúplex fundido, de gran interés tecnológico. El fenómeno de formación de fase sigma en esta aleación ocurre con gran efectividad en el rango de temperaturas de $1.073 \mathrm{~K}$ a $1.173 \mathrm{~K}$, aumentando la cantidad relativa del compuesto con la temperatura y tiempo de tratamiento térmico, hasta alcanzar valores cercanos a la saturación, después de $96 \mathrm{~h}$ de calentamiento. De esta forma, las mayores concentraciones de fase sigma a $1.073 \mathrm{~K}$, $1.123 \mathrm{~K}$ y $1.173 \mathrm{~K}$, determinadas mediante metalografía cuantitativa, se encuentran en la zona afectada térmicamente $(33 \%, 38 \%$, y $42 \%$ respectivamente, ) y, en menor proporción, en el metal base ( $31 \%, 35 \%$ y $39 \%$ respectivamente) y en la zona de fusión ( $30 \%, 31 \%$ y $27 \%$ ). El compuesto se forma por descomposición de la fase ferrítica en los bordes de grano ferrita-austenita, para luego extenderse hacia las zonas de ferrita, creando además, nuevas regiones de austenita secundaria. El mecanismo de crecimiento obedece a la ecuación de Jonson-Mehl-Avrami, la cual se ajusta con muy buena exactitud a los datos experimentales logrados en esta investigación. Finalmente, el contenido de ferrita decae hasta concentraciones prácticamente nulas.

\section{Agradecimientos}

Los autores expresan sus agradecimientos a la Universidad de Santiago de Chile (USACH) y al Fondo Nacional de Desarrollo de la Investigación Científica y Tecnológica (FONDECYT), Proyecto $N^{o} 1085053$, por el importante apoyo institucional y financiero otorgado para la realización del pre- sente proyecto. De igual forma, se reconoce el apoyo técnico prestado por la Srta. Gladys Olivares R., por la Sra. Alicia Durán C. y por el Sr. Felipe Gutiérrez P.

\section{REFERENCIAS}

[1] R.N. Gunn, Duplex Stainless, Steels, Ed. Abington Publishing, Cambridge, Inglaterra, 1997, pp. 1-13.

[2] J.R. Davis, Stainless Steels, Ed. ASM International, Metals Park, EE.UU., 1994, pp. 32-34.

[3] G. Herbsleb, Werkst. Korros. 33 (1982) 334-340.

[4] A. Ureña, E. Otero, M. V. Utrilla y C. J. Múñez, J. Mater. Proc. Tech. (2007) 624-631.

[5] S.D. Brandi, Mater. Sci. Forum 426 (2003) 4063-4068.

[6] D.N. Noble, Welding, Brazing, and Soldering, Ed. ASM International, Metals Park, EE.UU., 1993, pp. 471-481.

[7] M.R. EL Koussy, I.S. Mahallawi, W. Khalifa, M.M. AL Dawood y M. Bueckins, Mater. Sci. Tech. 20 (2004) 375-381.

[8] L. Karlsson, L. Ryen y S. Pak, Weld. J. 74 (1995) S28-S40.

[9] S. Hertzman, ISIJ Intern. 41 (2001) 580-589.

[10] R. Badji, M. Bouabdallah, B. Bacroix, C. Kahloun, K. Bettahar y N. Kherrouba, Mater. Charact. 59 (2008) 447-453.

[11] M. Pohl, O. Stortz y T. Glogwski, Mater. Charact. 58 (2007) 65-71.

[12] I. Calliari, M. Zanesco y E. Ramous, J. Mater. Sci. 41 (2006) 7643-7649.

[13] M. Pohl y O. Stortz, Z. Metallkd. 95 (2004) 631-638.

[14] A. K. Sihna, Prog. Mater. Sci. 15 (1972) 104-109.

[15] H. L. Yaquel, Acta. Crystallogr. B39 (1983) 20-28.

[16] H. L. Yaquel, Acta. Crystallogr. B39 (1983) 28-33.

[17] D. M. Escriba, E. Materna-Morris, R. L. Plaut y A. F. Padilha, Mater. Caract. 60 (2009) 1.214-1.219.

[18] G. Fargas, M. Anglada y A. Mateo, J. Mater. Proc. Tech. 209 (2009) 1.770-1.782.

[19] M. Martins y L.C. Casteletti, Mater. Caract. 55 (2005) 225-233.

[20] M.E. Wilms, V.J. Gadgil, J.M. Krougman y B.H. Kolster, Mater. High Temp. 9 (1991) 160-166.

[21] L. Jianchum, W. Tijan y Y. Riquier, Mater. Sci. Eng. 174 (1988) 149-166.

[22] Y.S. Sato, H. Kokawa y T. Kuwana, Sci. Tech. Weld. Join. 4 (1999) 41-49.

[23] J.O. Nilson, Mater. Sci. Tech. 8 (1992) 685-700. 
[24] American Society for Testing and Materials. ASTM A 890/A 890-99. Standard Specification for Casting, Iron-Chromium-NickelMolybdenum Corrosion-Resistant, Duplex (Austenitic/Ferrite) for General Application. ASTM International, 1999, pp. 1-4.

[25] American Society for Testing Materials. ASTM A 800/A 800 M-10. Standard Practice for Steel Casting, Austenitic Alloy, Estimating Ferrite
Content Therof. ASTM International, 1999, pp. 1-7.

[26] G.F. Vander Voort y G.M. Lucas, Metallography and Microstructures, Ed. ASM International, Metals Park, EE.UU., 2004, pp. 675-676.

[27] R. W. Cahn y P. Haasen, Physical Metallurgy, Ed. North Holland, Amsterdam, Holanda, 1996, pp. 1.435-1.436. 